\title{
CASE
}

or

EXTENSIVE

\section{INFLAMMATION AND OBSTR.UCTION}

OF THE VEINS

or .

THE RIGHT INFERIOR EXTREMITY,

ACCOMPANIED BY A SWOLLEN STATE OF THE LIMB.

The SUbJect, A pHThisical youth.

By T H. HOLBERTON, Esq. M.R.C.S.

\section{Read March 9th, 1830.}

FEB. 26th, 1830. Robert Willis, æet. 17, No. 3, Providence Court, North Audley Street, has laboured for ten months under pulmonary consumption, in the last stage of which he at present lies, extremely emaciated; with a hurried pulse, hectic fever, copious night sweats, purulent expectoration and diarrhœa, which, together with pain in the abdomen, has been very distressing during the last eight weeks.

Three weeks ago he was seized with pain in the right ham, and immediately afterwards a hot pain- 
ful swelling of the whole limb, and a considerable diminution of its muscular power, took place. At present the limb is two thirds larger than the left, hot and painful. It is colourless, excepting at the groin and inner side of the knee, where the superficial veins are enlarged and distended. The thigh pits when rather firmly pressed; is hotter and more painful along the course of the crural vessels than at its outer side; the femoral vein is clearly traced, like a hard cord, from the groin to where it perforates the tendon of the triceps; and the saphena at its upper part gives a similar sensation to the touch.

The right foot and ancle are very œdematous, and the leg also pits on pressure. There are great heat and tenderness in the hypogastric region, and especially in the right inguinal. Here a greater number of superficial veins are enlarged than on the opposite side, on which also, indeed, some are preternaturally distended. Half a dozen leeches were ordered to the right inguinal region. The wounds bled profusely for some hours, and afforded relief.

27th. The patient is easier in the lower part of the abdomen, and in the right thigh and leg. He can bear pressure rather better along the course of the femoral vessels than yesterday. The anasarca of the foot is increased. Being weak and emaciated, he was unwilling to bear a repetition of the 
leeches, and, as the subsequent treatment consisted only of emollient and anodyne applications, it is unnecessary to enter into detail. He died on Friday morning, 5th inst. (March) three o'clock.

\section{Examination,}

On the afternoon of the same day. Present, Dr. Ley, of Half-Moon Street, and Dr. Robert Lee, of Golden Square, the latter of whom was kind enough to visit the patient with me on the 26th ult.

The lungs were found extensively diseased. The veins from the middle of the inferior cava throughout the course of the right femoral, together with the left common iliac, were removed. Externally they were in parts knotty and hard and variously coloured, viz. whitish, dark, greyish, \&c. The density of the cellular membrane around each vein corresponded with the quantity of lymph effused into the cavity of the vessel. The arteries in contact with these veins were adherent to them in the same proportion.

Internal Appearance of the Veins.-In the vena cava for two inches above its bifurcation coagulable lymph, a few drops of puriform reddish matter, and an adventitious membrane, were found; the latter adherent to the inner surface of the vein, which, on the removal of the membrane, presented roughened bloody points. The voL. XVI. 
coats were thickened where they contained the membrane, but healthy above this part.

The right common iliac vein was affected in a similar manner to the inflamed portion of the vena cava, but more intensely, being more loaded with coagulum and having its coats thicker.

The same may be observed of the right external iliac. It contained more adventitious membrane, and its coats were thicker than those of the right common iliac; and the right femoral and its branches, particularly those about the groin, were even, if possible, more distended than the right external iliac.

At the mouth of the right internal iliac, and an inch and a half down the vessel, a firm coagulum and false membrane were found, most materially obstructing, if not entirely preventing, the circulation. Yet this vein did not seem so completely blocked up as those already mentioned, neither were its coats by any means so thick as theirs, though, where the coagulum was lodged, thicker than natural. In cutting off the vessel, about half an inch remained below this point, and was of bealthy appearance. The left common iliac and left external iliac were healthy. Serous fluid oozed from the incisions made in the thigh, the cellular membrane of which seemed denser than natural. 
The mucous membrane of the lower fifth of the small, and of the whole of the large intestines, was ulcerated. This altered condition of the bowel increased as it descended, and the greatest portion of the lining membrane of the rectum was destroyed.

The other abdominal viscera, the bones of the spine and pelvis, and their muscles, were healthy ; and no cause of the extensive mischief in the veins was discovered, unless we look to the rectum as the source of irritation:

To prove that diseased structure may give rise to inflammation of the neighbouring veins, Dr. Robert Lee, in his very valuable paper read before this Society and published in its Transactions, has related cases which have induced him to entertain that opinion, and has quoted very high authorities in support of it. "Thus," he says, " in a man who died of cancer of rectum in St. Bartholomew's Hospital, Mr. Lawrence observed the iliac veins inflamed and obstructed; and Laennec has stated, that it is not uncommon to find the veins in the neighbourhood of a cancerous breast filled with pus, either pure or mixed with blood, sometimes fluid, at other times more or less inspissated, and occasionally of the degree of consistence of an atheromatous tumour."

At the request of Dr. Robert Lee, I have en- 
deavoured to draw up a statement of the facts of the case, as they appeared to me; and I feel great pleasure in submitting them to the consideration of the Society, not presuming to offer an opinion of the cause of the disease of the veins, which I fear I have but too imperfectly described.

St. George's Infirmary, Mount Street, Grosvenor Square, . March 9, 1830.

\section{APPENDIX.}

Since the above paper was read to the Society, another case, very similar in its nature to that now related, has occurred to me, the leading points of which I may be allowed to state.

March 29, 1830. Isabella Weddell, æt. 35, a single woman. Ill sixteen months of tubercular phthisis. Added to the ordinary pectoral symptoms, she had tenderness over the whole of the abdomen, and acute pain at the left inguinal region. Catamenia absent during the last fourteen months. ?

On the 11th of May she was attacked by diarrhœa, attended with violent tenesmus. The motions were dark, slimy, and offensive, but unmixed with blood. She had from four to six discharges 
VEINS OF THE RIGHT INFERIOR EXTREMITY. 69

daily from this period, till her death, which took place on the 11th of June. The character of the stools varied but little from those described ; sometimes they contained flakes of mucus, at others they were more natural.

A few days after the attack of diarrhœa, pain extended from the left inguinal region to the lower extremity of the same side.

May 25. Left foot and ancle odematous; no difference in size between the two thighs; the heat of neither exceeds the natural standard, but there is acute pain in the situation of the femoral vein. From this time till her death, the cdema gradually, though slowly, extended up the whole of the limb. It felt " intolerably heavy." to the patient, but the inflammation of the vein was not.marked by a regular increase of heat, as sometimes the limb would be even colder than natural. The femoral vein, during the last fortnight of her life, was felt, like a hard cord, for three inches below Poupart's ligament.

P. M. Examination.-Present Mr. Alcock.

No tumour in the abdomen or pelvis was found to obstruct by pressure the functions of the absorbents of the left lower extremity.

Uterus, vagina, and bladder healthy. Mucous 
membrane of the small intestines natural ; that of the large intestines destroyed at different parts. Considerable ravages had taken place at the caput coli, but the ulcers gradually became less in number as we traced the intestinal canal towards its termination, so that on the mucous surface of the lowest four inches of the rectum four ulcerations only were found.

The vena cava and iliac veins of the right side were healthy. The left common iliac contained lymph adherent to the sides of the vessel. The same was observed at the union of the left internal iliac with the former vein; the remainder of the vessel was healthy in appearance. The left external iliac appeared healthy, except just at its lower portion, where it was completely blocked up by coagulum, adherent at one part only, but there firmly, and that part was a point immediately at and below the union of the two portions of a valve. The coagulum was found to extend along the two upper thirds of the femoral vein. The left hamorrhoidal veins were minutely dissected, and were in part healthy, while some contained phlebolites*. The vaginal, vesical, and

* "Calcareous concretions sometimes push the internal membrane before them, and descend with it into the cavity of the vein; the membrane becomes thin, and forms a true peduncle to the concretion. There is the closest analogy between these pedunculated concretions, and those which we sometimes find in the interior of joints. Like the latter; do the venous 
VEINS OF THE RIGHT INFERIOR EXTREMITY. 71

uterine veins were likewise carefully traced, and found to contain similar bodies. The right hæmorrhoidal veins were healthy; the right vaginal contained phlebolites.

Infirmary, Mount Street, Aug. 18, 1830.

concretions sometimes completely detach themselves from the coats of the vessel, and become loose in the cavity? Is this the origin of certain calcareous concretions found in the middle of clots which fill the cavity of these vessels? This is possible,-but it is also possible that these phlebolites may be produced in the blood itself.

"Phlebolites vary in size; some are scarcely so large as a millet-seed, others are equal to a small pea. We have found them in the dilated veins of the lower part of the rectum, neck of the bladder, uterus, ovaries, testicles, and in some of the subcutaneous veins of the lower extremities."-Précis d'Anatomie, Par G. Andral. Tome II., page 412. 